\title{
TIGHTLY CLOSED IDEALS
}

\section{MELVIN HOCHSTER AND CRAIG HUNEKE}

All rings are Noetherian, commutative, with 1 , and contain a field. We define the tight closure of an ideal in characteristic $p$ and for algebras essentially of finite type over a field, and use it to give new, much simpler proofs of many theorems in a greatly strengthened form, including the result that rings of invariants of linearly reductive groups acting on regular rings are CohenMacaulay (C-M), the Briançon-Skoda theorem, and the monomial conjecture. Although we do not define tight closure in arbitrary rings, many of our results can be proved in that generality by using Artin approximation to reduce to the affine case. Results discussed here are treated in full in $[\mathbf{H H}]$.

1. Tight closure. Let $R^{0}=R-\bigcup\{P: P$ is a minimal prime of $R\}$. Let $I \subseteq R$ be an ideal. If $\operatorname{char} R=p>0$ we say that $x \in R$ is in the tight closure, $I^{*}$, of $I$, if there exists $c \in R^{0}$ such that for all $e \gg 0, c x^{p^{e}} \in I^{\left[p^{e}\right]}$, where $I^{[q]}=\left(i^{q}: i \in I\right)$ when $q=p^{e}$. If $R$ is of finite type over a field $K$ of characteristic 0 , we say that $x \in I^{*}$ if there exist $c \in R^{0}$, a finitely generated Z-subalgebra $D \subseteq K$, a finitely generated $D$-flat $D$-subalgebra $R_{D}$ of $R$, and an ideal $I_{D} \subseteq R_{D}$ such that $R \cong K \otimes_{D} R_{D}, I=I_{D} R$, and for all maximal ideals $m$ of $D$, if $\kappa=R / m$ (with the subscript $\kappa$ denoting images after applying $\kappa \otimes_{D}$ ), and $p=\operatorname{char} \kappa$, then $c_{\kappa} x_{\kappa}^{q} \in I_{\kappa}^{[q]}$ (in $R_{\kappa} \cong R_{D} / m R_{D}$ ) for all $q$ of the form $p^{e}, e \gg 0$. If $R$ is essentially of finite type over a field $K$ of char 0 we define $I^{*}$ as $\bigcup_{B}(I \cap B)^{*}$ as $B$ runs through all subrings of $R$ of finite type over $K$ such that $R$ is a localization of $B$.

These intricate definitions yield an immensely powerful tool. We note that $I \subseteq I^{*}=I^{* *}$ and that $I^{*} \subseteq \bar{I}$, the integral closure of $I$, but that $I^{*}$ is usually much smaller than $\bar{I}$. If $I=I^{*}$, we call $I$ tightly closed. A key point is that if $R$ is regular, then $I=I^{*}$ for all $I$. Suppose that $R$ is regular of $\operatorname{char} p>0$. To see that $I=I^{*}$ we may assume that $(R, m)$ is local and that $y \in I^{*}-I$ where $I=\left(x_{1}, \ldots, x_{m}\right) R$. Then for some $c \in R^{0}, c y^{q} \in I^{[q]}$ for all $q=p^{e} \gg 0$. Since the Frobenius $f$ is flat, $I^{[q]}: y^{q}=(I: y)^{[q]}$, which implies that $c \in m^{q}$, and since this is true for every $q \gg 0, c=0$, a contradiction. We call rings such that in all localizations $I=I^{*}$ for all $I F$-regular.

(1.1) REMARK. If $R \subseteq S$ are domains where * is defined, $S$ is regular, and $I$ is an ideal of $R$ then $I^{*} S=I S$, since $I S$ is tightly closed in $S$. Hence, $I^{*}$ is contained in all ideals containing $I$ which are contracted from regular overrings. We do not know whether $I^{*}$ is the intersection of all ideals containing $I$ which are contracted from regular overrings.

Received by the editors August 13, 1987.

1980 Mathematics Subject Classification. Primary 13C99.

Both authors were supported in part by NSF grants. 
(1.2) REMARK. A Gorenstein local ring has the property that $I=I^{*}$ for all $I$ iff the ideal generated by a single s.o.p. (system of parameters) is tightly closed.

2. Subrings of regular rings. It is not hard to prove that $F$-regular rings are normal. Of much greater significance is:

(2.1) THEOREM. An F-regular ring which is module-finite over a regular ring is Cohen-Macaulay.

This is immediate from the following crucial fact:

(2.2) THEOREM. Let $R$ be a module-finite and A-torsion-free overring of $A$, where $A$ is a regular domain of char $p>0$ or else essentially of finite type over a field, and let $x_{1}, \ldots, x_{n}$ be elements of $A$ which are part of a s.o.p. in $R_{P}$ for all $P$ which contain them. Let $I=\left(x_{1}, \ldots, x_{n-1}\right) R$. Then $I:_{R} x_{n} R \subseteq I^{*}$.

SKETCH OF PROOF IN char $p$. One reduces to the case where the $x_{i}$ are an $A$-sequence. One can choose a free $A$-module $F \subseteq R$ such that $R / F$ is killed by $c \in A-\{0\}$. If $y x_{n} \in \sum_{i=1}^{n-1} R x_{i}$, raising to the $q$ th power where $q=p^{e}$ and multiplying by $c$ yields $x_{n}^{q}\left(c y^{q}\right) \in \sum_{i=1}^{n-1} x_{i}^{q} F \Rightarrow c y^{q} \in\left(x_{1}^{q}, \ldots, x_{n-1}^{q}\right) F \subseteq$ $I^{[q]} \Rightarrow y \in I^{*}$. (Cf. [H1, Lemma 4.4].)

It is trivial to prove that if $R \subseteq S$ are domains, $J=J^{*}$ in $S$ and $I=J \cap R$, then $I=I^{*}$ in $R$. In consequence:

(2.3) THEOREM. If $R \subseteq S$ and $R$ is a direct summand of $S$ as an $R$ module, then if $S$ is $F$-regular, $R$ is $F$-regular and so $C$ - $M$.

Let $x_{1}, \ldots, x_{d}$ be a s.o.p. for the local ring $R$. Let $I_{r}=\left(x_{1}, \ldots, x_{r}\right) R$. If tight closure is defined in $R$ then, under mild restrictions, in order that $R$ be C-M it suffices that $I_{d}$ (or $I_{d-1}$ ) be tightly closed. When $R$ is a subring of a regular ring $S$ one can then deduce the C-M property for $R$ from the contractedness of $I_{d}$ (or $I_{d-1}$ ) from $S$. The condition that one ideal generated by a s.o.p. be contracted is enormously weaker than the condition that $R$ be a direct summand: it holds frequently in char $p$ when the direct summand condition does not.

Even when ${ }^{*}$ is not defined, Artin approximation yields:

(2.4) THEOREM. Let $R$ be an equicharacteristic ring which is a direct summand as an $R$-module (or a pure subring: cf. [HR1]) of a regular ring $S$. Then $R$ is $C-M$.

A result similar to (2.4) was first proved in [HR1] for rings of invariants of linearly reductive groups acting on regular rings. The result was extended to summands of regular rings for algebras of finite type in $[\mathbf{K}]$, while in $[\mathbf{B}]$ it was shown that in the affine and analytic cases direct summands of rational singularities are rational. None of these earlier arguments permitted extension to the general equicharacteristic case.

One of the most important applications of tight closure is to calculating the effect of ideal operations like intersection, colon, etc. on ideals generated by 
monomials in a system of parameters (and quite a bit more generally). There is an expected or "formal" answer which is what one would get if the elements formed an $R$-sequence. The key point is that under mild hypotheses the answer obtained is contained in the tight closure of the expected answer, and hence, by Remark (1.1), coincides with it if one expands to a regular overring. This last statement does not refer to tight closure and can be proved in great generality in char 0 . In fact one can establish much more general statements, of which the following very powerful result on vanishing of maps of Tor is an example.

(2.5) VANISHING THEOREM. Let $A \rightarrow R \stackrel{f}{\rightarrow} S$ be maps of equicharacteristic rings such that $A, S$ are regular domains, $R$ is a module-finite overring of $A$, and $f$ is injective. Assume that $R$ is excellent. Let $M$ be any A-module of finite type. Then for all $i \geq 1$, the map $\operatorname{Tor}_{i}^{A}(M, R) \rightarrow \operatorname{Tor}_{i}^{A}(M, S)$ is zero.

Note that there is no finiteness condition on the map $f$. The case where $M=A /\left(x_{1}, \ldots, x_{i}\right)$, where the $x_{t}$ are a s.o.p. in $A$, and $R$ is a direct summand of $S$, implies that summands of regular rings are C-M. The case where $M=$ $A / J$ and $S$ is a DVR dominating the local ring $R$ implies the canonical element conjecture [H2]. The proof of (2.5) uses the notion of the tight closure of a submodule of a module: one shows that certain cycles are in the tight closure of the boundaries and hence are boundaries once one passes to the regular ring $S$, where every submodule is tightly closed. (In char $p$, if $N \subseteq M$ we say $y \in M$ is in $N^{*}$ if there exists $c \in R^{0}$ such that for all $e \gg 0, c(1 \otimes y)$ maps to 0 in $F^{e}(M / N)$, where $F$ is the Peskine-Szpiro functor [PS, p. 330, Definition (1.2)].)

It would evidently be of great importance if the theory of tight closure could be extended to mixed characteristic.

3. The Briançon-Skoda theorem and rational singularities. There is a strong connection between rational singularities and $F$-regularity. We observe:

(3.1) THEOREM. Suppose that $R$ is of finite type over a field $K$ of char 0 and that $I=I^{*}$ for all $I \subseteq R$. If either: (a) $R$ has isolated singularities or (b) $R$ is $\mathbf{N}$-graded with $R_{0}=K, m=\bigoplus_{i=1}^{\infty} R_{i}$, and $R$ has rational singularities except possibly at $m$, then $R$ has rational singularities.

In particular, $F$-regular surfaces have rational singularities. The converse is not true: an example of [W] shows that a surface in char 0 may have rational singularities without even being of $F$-pure type in the sense of [HR2]. The proof of part (a) depends on the following result, which generalizes a theorem of Briançon-Skoda first proved analytically [BS] and later algebraically (see $[\mathbf{L T}, \mathbf{L S}])$.

(3.2) THEOREM. Let $R$ be a ring of char $p>0$ or an algebra essentially of finite type over a field and let $I$ be an ideal of height at least one generated by $n$ elements. Then $\overline{I^{n}} \subseteq I^{*}$. In particular, if $R$ is F-regular then $\overline{I^{n}} \subseteq I$.

PROOF IN char $p$. Let $I=\sum_{i=1}^{n} x_{i} R$ and $a=I^{n}$. If $a$ is contained in the union of $I^{*}$ and the minimal primes of $R$ it must be $\subseteq I^{*}$. If not 
choose $y$ in $a$ not in any minimal prime of $R$. One can show that $y^{k+m} \in$ $a^{m+1}(a+y R)^{k-1} \subseteq a^{m}$ for a certain integer $k \geq 1$ and all $m \in \mathbb{N}$. Let $c=y^{k}$, $m=q=p^{e}$ and note that $a^{q}=I^{n q} \subseteq I^{[q]}$ (since $I$ has $n$ generators), i.e. $c y^{q} \in I^{[q]}$ for all $q$.

\section{REFERENCES}

[B] J.-F. Boutot, Singularités rationelles et quotients par les groupes réductifs, Invent. Math. 88 (1987), 65-68.

[BrS] J. Briançon and H. Skoda, Sur la clôture intégrale d'un idéal de germes de fonctions holomorphes en un point de $C^{\infty}$, C.R. Acad. Sci. Paris Sér. A 278 (1974), 949-951.

[GR] H. Grauert and O. Riemenschneider, Verschwindungsätze für analytische kohomologiegruppen auf komplexen Räumen, Invent. Math. 11 (1970), 263-290.

[H1] M. Hochster, Topics in the homological theory of modules over commutative rings, C.B.M.S. Regional Conf. Ser. in Math., No. 24, Amer. Math. Soc., Providence, R.I., 1975.

[H2] Canonical elements in local cohomology modules and the direct summand conjecture, J. Algebra 84 (1983), 503-553.

[HH] M. Hochster and C. Huneke, Tight closures, invariant theory, and the Briançon-Skoda Theorem, in preparation.

[HR1] M. Hochster and J. L. Roberts, Rings of invariants of reductive groups acting on regular rings are Cohen-Macaulay, Adv. in Math. 13 (1974), 115-175.

[HR2] _ , The purity of the Frobenius and local cohomology, Adv. in Math. 21 (1976), 117-172.

[K] G. Kempf, The Hochster-Roberts theorem of invariant theory, Michigan Math. J. 26 (1979), 19-32.

[LS] J. Lipman and A. Sathaye, Jacobian ideals and a theorem of Briançon-Skoda, Michigan Math. J. 28 (1981), 199-222.

[LT] J. Lipman and B. Teissier, Pseudo-rational local rings and a theorem of BriançonSkoda about integral closures of ideals, Michigan Math. J. 28 (1981), 97-116.

[PS] C. Peskine and L. Szpiro, Dimension projective finie et cohomologie locale, I.H.E.S. Publ. Math. 42 (1973), 323-395.

[W] K. Watanabe, Study of F-purity in dimension two, Tokai University, Hiratsuka, Japan, preprint.

Department of Mathematics, University of Michigan, Ann ARbor, MICHIGAN 48109-1003

Department of Mathematics, Purdue University, West lafayette, INDIANA 47907 